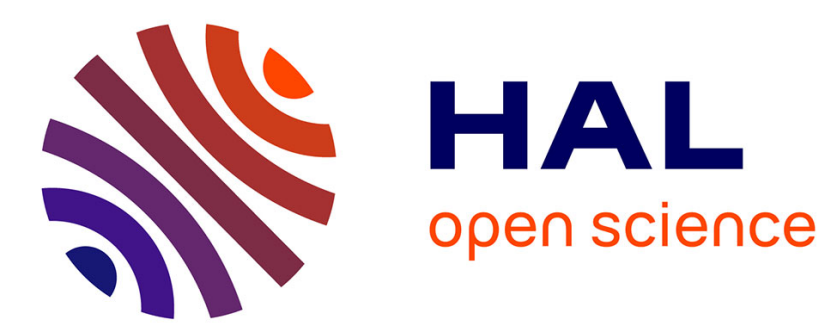

\title{
Emploi des liants pour le pressage des aliments des animaux : aspects technologiques et nutritionnels
}

\author{
J.P. Melcion
}

\section{To cite this version:}

J.P. Melcion. Emploi des liants pour le pressage des aliments des animaux: aspects technologiques et nutritionnels. Productions Animales, 1995, 8 (2), pp.83-96. hal-00896106

\section{HAL Id: hal-00896106 https://hal.science/hal-00896106}

Submitted on 1 Jan 1995

HAL is a multi-disciplinary open access archive for the deposit and dissemination of scientific research documents, whether they are published or not. The documents may come from teaching and research institutions in France or abroad, or from public or private research centers.
L'archive ouverte pluridisciplinaire HAL, est destinée au dépôt et à la diffusion de documents scientifiques de niveau recherche, publiés ou non, émanant des établissements d'enseignement et de recherche français ou étrangers, des laboratoires publics ou privés. 
INRA Prod. Anim., $1995,8(2), 83-96$

\section{J.P. MELCION}

INRA Laboratoire de Technologie Appliquée à la Nutrition, BP 1627, 44316 Nantes Cedex 03
Emploi des liants pour le pressage des aliments des animaux : aspects technologiques et nutritionnels

\begin{abstract}
Les adjuvants de pressage sont utilisables pour l'agglomération des aliments, en raison de leurs propriétés liantes, assurant la cohésion du granulé, et lubrifiantes, réduisant la consommation d'énergie électrique de la presse. Ils n'ont généralement pas de valeur nutritionnelle propre, mais peuvent modifier les paramètres de la digestion.
\end{abstract}

Le pressage dans la fabrication d'un aliment composé est une opération dont le résultat est encore aléatoire : le mode d'action des forces qui régissent l'association des particules entre elles est en grande partie incon-

\section{Résumé}

\begin{abstract}
Les liants (ou adjuvants de pressage) sont des additifs généralement dépourvus de valeur alimentaire qui sont incorporés à faible taux $(0,5$ à $2,5 \%)$ dans les aliments composés dans le seul but d'augmenter le rendement des presses et la cohésion des granulés. Ces substances peuvent être d'origine organique ou minérale. Parmi les substances organiques, les lignosulfites sont des sous-produits de l'industrie papetière. Les substances minérales appartiennent essentiellement à la famille des argiles. D'autres additifs tels que les polysaccharides peuvent être utilisés dans l'élaboration des aliments pour les animaux aquatiques.
\end{abstract}

Il existe des tests de laboratoire susceptibles d'évaluer l'aptitude liante ou lubrifiante de ces adjuvants de pressage, en fonction principalement de l'humidité et du type de matière première. Les essais à échelle pilote montrent généralement une influence positive de l'addition des lignosulfites sur les propriétés mécaniques des agglomérés et une tendance à une réduction de l'énergie consommée par la presse dans le cas de mélanges riches en céréales (et en amidon). L'effet des substances minérales est assez variable en regard des conditions de pressage. A échelle industrielle, les petites différences observées peuvent être masquées par les variations aléatoires liées aux essais en usine.

Les adjuvants de pressage n'ont pas de valeur nutritionnelle en soi. Les lignosulfites et autres composés organiques peuvent faire exception en raison de leur teneur - bien que limitée - en sucres simples. Les liants minéraux sont considérés usuellement comme des diluants de la ration. Les effets indirects constatés sur l'indice de consommation peuvent être dûs à une augmentation de dureté des granulés. Un effet de tannage des protéines de l'aliment est possible avec les lignosulfites. Cependant, la structure et le comportement physique des argiles (bentonite, sépiolite) peuvent expliquer certains effets sur la digestibilité de l'énergie chez le monogastrique, par une modification de la rétention d'eau et de la durée de transit dans l'intestin de l'animal, et sur l'utilisation de l'azote chez le ruminant. nu. Produire des granulés de cohésion suffisante à bas coût demande un équilibre entre les matières premières utilisées, les caractéristiques des matériels, leur mise en cuvre opératoire, et l'addition possible d'adjuvants connus pour leurs propriétés liantes et/ou lubrifiantes (Olentine 1980).

Ces propriétés liantes / lubrifiantes peuvent provenir des matières premières utilisées principalement en raison de leur contribution nutritionnelle - mélasses, amidons gélatinisés, glycérides du suif ou sels d'acides gras - ou bien d' additifs particuliers, les adjuvants de pressage, qui sont incorporés prioritairement pour leurs propriétés liantes et/ou lubrifiantes, et secondairement pour leur teneur en éléments nutritifs et les modifications nutritionnelles qu'ils peuvent induire.

Il nous faut à cet égard établir une distinction entre pouvoir liant et pouvoir lubrifiant. Un " lubrifiant " facilite le glissement des particules entre elles et influe directement sur le rendement de l'agglomération, alors qu'un " liant " tend plutôt à cimenter les particules, donc à favoriser la cohésion de l'aggloméré. En pratique, le terme "liant " couvre ces deux aspects parfois opposés.

\section{1 / Origines et propriétés des adjuvants de pressage}

Les adjuvants de pressage sont, pour la plupart, soit des sous-produits d'origine industrielle, soit des produits d'extraction 
divers, que l'on peut diviser un peu arbitrairement en deux groupes principaux : les substances d'origine minérale d'une part, d'origine organique d'autre part.

\section{1 / Adjuvants d'origine minérale}

\section{a / Argiles à structure lamellaire}

Les argiles sont des silicates d'aluminium hydratés, comprenant des atomes de silice et d'aluminium en majeure partie, disposés selon une structure en feuillets constitués de mailles cristallines (phyllosilicates). Par suite des substitutions ioniques dans la maille du minéral, chaque grain d'argile est chargé d'électricité négative sur sa surface et les feuillets qui le composent sont plus ou moins reliés fortement les uns aux autres. Chaque grain d'argile est enveloppé d'un film d'eau de nature spéciale (eau adsorbée) et, en fonction des cations associés, de l'eau intersticielle peut s'ajouter à la précédente et venir s'insérer entre les feuillets. Cette structure suggère un effet lubrifiant par le glissement des feuillets l'un sur l'autre. Lorsque la teneur en eau diminue, les particules se rapprochent, chassant l'eau intersticielle puis l'eau adsorbée pour établir des ponts solides. Ainsi, en présence d'eau ou de vapeur, les argiles seraient capables d'une part de prévenir le blocage des presses, et d'autre part de cimenter les particules après refroidissement (et séchage) par un effet de rétraction des feuillets. On trouve, dans ce groupe, des produits pulvérulents (tableau 1), dont la couleur va du gris au blanc, et dont les appellations commerciales ne recouvrent pas toujours les noms minéralogiques véritables.

La clé de l'utilisation des argiles comme adjuvants est la présence de quantités d'eau adéquates dans le mélange argile-aliment. L'agglomération (ou pressage ou encore " granulation ") intervient à la fin de la fabrication d'un aliment composé. Elle consiste à com-

Figure 1. Schéma d'une presse et principe de la compaction dans une filière de presse (David et Lefumeux 1973).

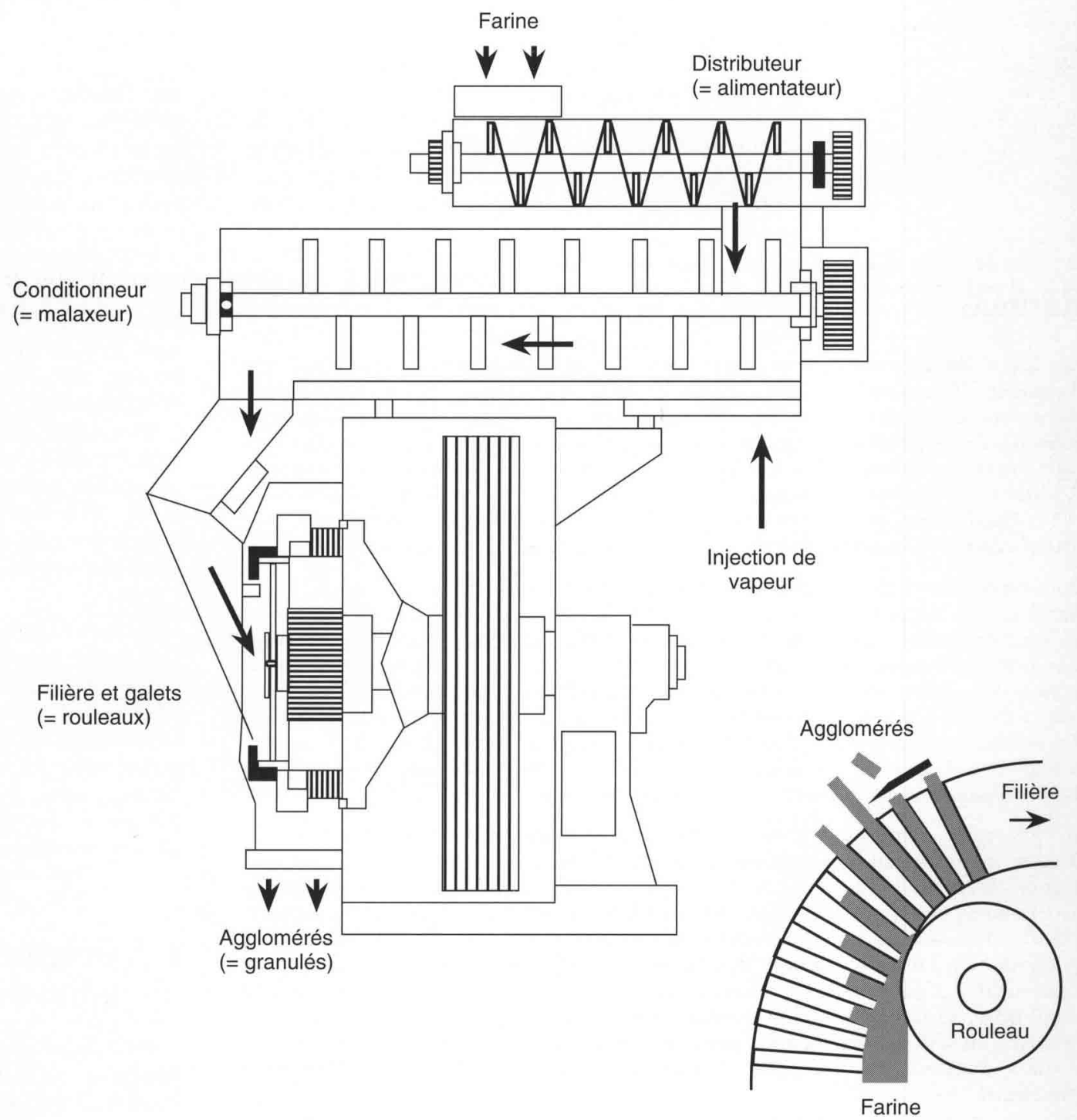


pacter le mélange dans une presse (figure 1), après généralement injection de vapeur vive dans la masse à l'aide d'un conditionneur (David et Lefumeux 1973). Comme les argiles n'absorbent pas l'eau liée qui se trouve dans l'aliment, celle-ci est apportée par la condensation de la vapeur dans le conditionneur, ou par apport direct en amont de la presse dans la mélangeuse, ou par la mélasse. Une basse pression de vapeur est généralement recommandée, de manière à favoriser une condensation plus importante dans le conditionneur. En se fondant sur les pratiques cimentières, l'idéal serait d'ajouter l'argile après humidification de l'aliment qui adsorbe l'eau et agit comme un concurrent. Une filière dite "peu comprimante ", dont le rapport longueur de canal / diamètre de canal est inférieur à 10 est également recommandée en présence d'argile, ce qui permet d'utiliser davantage de vapeur sans bloquer le fonctionnement de la presse. Les taux d'incorporation sont généralement de 1 à $2 \%$.

\section{Bentonites}

Les bentonites (Fort-Benton, USA) sont parmi les premiers liants apparus en alimentation animale. Les bentonites contiennent plus ou moins de montmorillonite (Montmorillon, Vienne, France), qui est une argile à 3 feuillets associée à des composés colloïdaux. Plus la teneur en montmorillonite est importante, plus élevées sont les propriétés lubrifiantes. Les feuillets sont liés par des ponts ioniques dûs à la présence de cations. La liaison est si faible qu'elle se brise sous la simple action de l'eau (Caillère et al 1982). La capacité d'échange est élevée et décroît dans l'ordre suivant $\left(\mathrm{Ca}^{++}, \mathrm{Mg}^{++}, \mathrm{K}^{+}, \mathrm{H}^{+}, \mathrm{Na}^{+}\right)$. On utilise le terme bentonite de sodium ou bentonite de calcium en fonction de la nature de l'ion échangeable. La bentonite de sodium a généralement une plus grande capacité à se lier à l'eau que la bentonite de calcium. A l'état pur, elle peut absorber au moins 5 fois sa masse d'eau. Lorsqu'elle est totalement saturée, elle expanse : un gel est obtenu, qui perd sa plasticité et se disperse sans former de pâte liante. La bentonite de calcium par contre n'absorbe qu'une à une fois et demie sa masse d'eau (Olentine 1980).

\section{Kaolinites}

Les kaolinites sont également des argiles en feuillets. Leur structure ( 2 feuillets) et leur composition globale sont semblables à celle des bentonites (tableau 1), mais les feuillets sont liés étroitement par des liaisons hydrogène - d'où une capacité d'échange très faible (tableau 2) qui les rendent incapables de se gonfler dans l'eau et de donner naissance à des gels. Une faible surface spécifique et l'absence de charges entraînent une basse capacité d'échange. D'autres argiles comme la muscovite ou l'attapulgite peuvent également se trouver associées à la kaolinite, avec des comportements similaires.

\section{Vermiculites}

La vermiculite est une roche formée de couches micacées séparées par deux molécules d'eau contenant des ions hydratés, qui assurent de faibles forces de liaison entre feuillets. La capacité d'échange et les propriétés d'absorption sont importantes et peuvent être accrues par expansion à la chaleur à 800 "C. Ces argiles peuvent aussi être utilisées pour absorber des liquides tels que la mélasse, le chlorure de choline ou l'urée.

\section{b / Argiles à structure tubulaire}

\section{Sépiolite}

La sépiolite appartient à une famille d'argiles (Alvarez 1984) dont les feuillets sont orientés de manière à modifier le faciès lamellaire classique en lui faisant prendre une structure tubulaire ("minéral à pseudofeuillets "). La structure d'ensemble est alors comparable à une brique creuse (Wolter et al 1990). La section de ces micro-canaux, dans lesquels des liquides et des gaz comme l'eau ou l'ammoniac peuvent s'insérer, est de $0,38 \mathrm{~nm}^{2}$. Ces canaux sont à l'origine de la surface spécifique élevée (plus de $300 \mathrm{~m}^{2} / \mathrm{g}$ ) et de la capacité d'absorption ( 1 à 1,5 fois son poids d'eau) du minéral. Cette argile est riche en magnésium (tableau 1) car il y a remplacement partiel de l'aluminium par le magnésium. Sa capacité d'échange ionique est faible (tableau 2).

Par ses mécanismes d'action, la sépiolite peut être classée dans le même groupe d'adju-

Tableau 1. Composition (en \% produit brut*) des produits d'origine minérale (sources diverses).

\begin{tabular}{|l|c|c|c|c|c|}
\hline & Bentonite & Kaolinite & Vermiculite & Sépiolite & $\begin{array}{c}\text { Aluminate } \\
\text { de calcium }\end{array}$ \\
\hline $\begin{array}{l}\text { Perte au feu } \\
\left(5500^{\circ} \mathrm{C}\right)\end{array}$ & 8 à 17 & 11 à 14 & & & \\
$\mathrm{SiO}_{2}$ & 58 à 69 & 43 à 52 & 45 & 61 & 3 à 5 \\
$\mathrm{Al}_{2} \mathrm{O}_{3}$ & 15 à 21 & 32 à 39 & 30 & 5 & 38 à 40 \\
$\mathrm{Fe}_{2} \mathrm{O}_{3}$ & 2 à 5 & 1 à 3 & 9 & 2 & 14 à 17 \\
$\mathrm{TiO}_{2}$ & $<0.5$ & 2 à 3 & 20 & 19 & 2 à 3 \\
$\mathrm{MgO}$ & 2 à 5 & $<0,5$ & 1 & 1 & 37 à 39 \\
$\mathrm{CaO}$ & 1 à 4 & $<0,5$ & 1 & & \\
\hline
\end{tabular}

* humidité $<1 \%$ du produit brut.

Les argiles ont une structure en feuillets qui peuvent glisser l'un sur l'autre lorsque la teneur en eau est suffisante. 
Tableau 2. Propriétés physiques des adjuvants minéraux.

\begin{tabular}{|l|c|c|c|c|c|}
\hline & Bentonite & Kaolinite & Vermiculite & Sépiolite & $\begin{array}{c}\text { Aluminate } \\
\text { de calcium }\end{array}$ \\
\hline $\begin{array}{l}\text { Surface spécifique* } \\
\left(\mathrm{m}^{2} / \mathrm{g}\right)\end{array}$ & & $\begin{array}{c}20(\mathrm{BET}) \\
1,5(\mathrm{Blaine})\end{array}$ & & $300(\mathrm{BET})$ & \\
Densité apparente $\left(\mathrm{g} / \mathrm{cm}^{3}\right)$ & 1 & 1,1 à 1,24 & 0,1 & 0,54 à 0,6 & 3 (Blaine) \\
$\begin{array}{l}\text { Densité vraie }\left(\mathrm{g} / \mathrm{cm}^{3}\right) \\
\text { Absorption d'eau }(\%)\end{array}$ & 2,5 & 2,35 à 2,64 & & 2,0 à 2,3 & 1,15 \\
Capacité d'échange (méq/g) & 500 à 650 & 50 à 65 & 300 & 100 à 150 & 40 \\
\end{tabular}

* La méthode BET utilise l'adsorption gazeuse, la méthode Blaine la perméamétrie.

vants que les autres argiles: il a été démontré qu'une humidification correcte de la surface en vue de l'agglomération peut être apportée par 0,5 à 2,0\% d'eau. Son pouvoir absorbant peut être mis à profit dans des rations contenant des taux élevés de matières grasses (Lopez et Alvarez 1991) pour en accroître la durabilité.

\section{c / Aluminate de calcium}

L'aluminate de calcium est le résultat de la fusion de minerai d'aluminium mélangé à du carbonate de calcium à très haute température $\left(>1500^{\circ} \mathrm{C}\right)$ produit par l'industrie cimentière. Après un broyage très fin (100\% $<100 \mu \mathrm{m}$ ), il se présente comme une poudre foncée et de masse volumique élevée (tableau 2) qui contient environ $38 \%$ d'équivalent $\mathrm{CaO}$. La cristallisation du produit après hydratation (40\% en masse par rapport au produit sec) semble être la clé du comportement liant. La chaleur est généralement un catalyseur de la réaction de cristallisation, qui peut s'effectuer de manière lente et différée après incorporation dans un aliment aggloméré. L'enrobage des particules par la matière grasse dans certains aliments exerce, semble-t-il, un effet contraire. Le taux d'incorporation recommandé est de 0,6 à $1,0 \%$.

\section{2 / Adjuvants d'origine organique}

\section{a / Lignosulfites}

Les lignosulfites (ou lignosulfonates pour les anglo-saxons) constituent un groupe de substances dérivées de l'industrie papetière (Dumée et Michelin 1970). Les copeaux de bois sont traités en autoclave sous 8 à 9 bar de pression à haute température (150 à $162{ }^{\circ} \mathrm{C}$ ) par une solution aqueuse de bisulfite d'ammonium ou de calcium contenant de l'anhydride sulfureux libre. Pratiquement tous les composants ligneux du bois sont solubilisés pour ne laisser subsister que la cellulose. Il y a également hydrolyse partielle des hémicelluloses et solubilisation des composés résineux du bois. La solution est séparée par pressage et, après neutralisation par la soude ou la magnésie, la "liqueur»obtenue qui contient 50 à 55 de matière sèche est alors concentrée et séchée. Elle se présente commercialement sous forme de poudre brune très hygroscopique. Selon le procédé et le type de bois traité, la matière sèche contient 40 à $53 \%$ de lignine, 23 à $41 \%$ d'oligosaccharides, principalement des hexoses (fermentescibles) et des pentoses (non fermentescibles) 2 à $13 \%$ de matières minérales (Hogan 1974, Kivimäe 1978 : tableau 3). La teneur en soufre varie de 2 à $5 \%$.

La structure moléculaire de la lignine sulfonée n'est pas bien connue. La présence d'un ion sulfonate chargé négativement près de la surface de la molécule peut expliquer ses propriétés hydrophiles et tensio-actives (Chan et al 1976). Dans les aliments, ces propriétés expliquent l'effet lubrifiant des lignosulfites. Leur viscosité, associée à celle des composés glucidiques, accroît les forces de liaison entre les particules d'aliment. Le taux d'incorporation recommandé est de 1 à $2,5 \%$.

De plus, les groupements aminés des protéines de l'aliment peuvent se combiner aux groupements sulfonates par une véritable

Tableau 3. Composition des liants dérivés du bois (\% matière sèche).

\begin{tabular}{|c|c|c|c|c|}
\hline & Lignine & $\begin{array}{c}\text { Hydrates } \\
\text { de carbone }\end{array}$ & $\begin{array}{c}\text { Sucres } \\
\text { réducteurs }\end{array}$ & Minéraux \\
\hline $\begin{array}{l}\text { Extrait d'hémicellulose } \\
\text { Lignosulfite de } \mathrm{NH}_{4} \\
\text { - neutre } \\
\text { - acide } \\
\text { Lignosulfite de } \mathrm{Ca} \\
\text { - bois feuillus } \\
\text { - bois résineux }\end{array}$ & $\begin{array}{l}10 \text { à } 14 \\
41 \text { à } 45 \\
48 \text { à } 52 \\
40 \text { à } 45 \\
47 \text { à } 53\end{array}$ & $\begin{array}{l}80 \text { à } 84 \\
26 \text { à } 32 \\
23 \text { à } 27 \\
35 \text { à } 41 \\
24 \text { à } 30\end{array}$ & $\begin{array}{l}13 \text { à } 18 \\
1 \text { à } 5 \\
10 \text { à } 14 \\
16 \text { à } 30 \\
12 \text { à } 28\end{array}$ & $\begin{array}{c}4 \\
1 \text { à } 2 \\
1 \text { à } 2 \\
8 \text { à } 10 \\
10 \text { à } 13\end{array}$ \\
\hline
\end{tabular}


réaction de tannage. Un tiers de ces liaisons seraient irréversibles selon Gustavson (1956), principalement avec les lignines sulfonées de haut poids moléculaire.

\section{b / Extraits d'hémicellulose}

Les " extraits d'hémicellulose " sont des produits issus de la production des panneaux de particules de bois, disponibles essentiellement sur le marché nord-américain. Les copeaux de bois sont dilacérés par action de la vapeur à hautes pression et température, puis lavés à l'eau. Les produits solubles entraînés par les eaux de ruissellement sont recueillis, concentrés et séchés. L'extrait se présente sous forme d'une poudre brun clair qui contient 80 à $84 \%$ de polysaccharides et peu de lignine (tableau 3). Leur viscosité en présence d'eau peut expliquer leur effet liant dans un aliment.

\section{c / Celluloses substituées}

Les éthers de la cellulose (carboxy-méthylcellulose par exemple), couramment employés en industrie alimentaire comme agents épaississants ou gélifiants, ont été adaptés à l'alimentation animale. Plus le degré d'éthérification est élevé $(0,2$ à 0,8$)$, plus leur affinité pour l'eau est élevée et plus basse est leur viscosité en phase hydratée. Ils sont capables d'absorber l'eau en proportions très élevées jusqu'à se solubiliser. Ceci explique leurs propriétés filmogènes et lubrifiantes en présence d'une proportion d'eau suffisante ajoutée lors du conditionnement. Le taux d'incorporation recommandé est bas $(0,1 \%)$ en partie pour limiter les inconvénients physiologiques. Un effet inhibiteur sur l'hydrolyse par la pepsine de protéines pures a été mis en évidence (Valaris et Harper 1973).

\section{d / Gommes végétales}

Les "gommes" végétales sont des polysaccharides de haut poids moléculaire extraits d'algues (alginates, caraghénanes), ou de graines de légumineuses (guar, caroube). Elles sont traitées chimiquement pour accroître leur affinité pour l'eau ainsi que leurs pouvoirs gélifiant et épaississant. En raison de leur prix et de leur relative inefficacité en milieu peu hydraté, elles sont utilisées davantage en milieu humide (40-45\% d'eau) pour des applications particulières. Leur emploi est bénéfique dans les aliments pour poissons et crustacés en vue d'accroître leur stabilité à l'eau et de réduire la pollution des bassins d'élevage (Storebakken 1985, Meyers 1991). Elles peuvent également se trouver sur le marché en association avec d'autres composés (pâtes de neutralisation issues de l'huilerie).

\section{e / Mélanges}

Plusieurs types de mélanges, qui visent à combiner les propriétés des adjuvants simples décrits ci-dessus, sont commercialisés comme liants ou lubrifiants. L'un des plus connus est une association de carboxyméthyl-cellulose (CMC), d'hydrolysats protéiques et d'extraits de sassafras (lauracée de Chine et d'Amérique du Nord). Le taux d'incorporation du mélange est plus élevé que celui de la CMC pure $(1,25-2,5 \%)$. Les propriétés filmogène et lubrifiante de la CMC sont associées à l'odeur agréable de petites quantités de sassafras. L'eau et la température sont, là aussi, essentielles à une pleine efficacité du mélange, mais cette efficacité décroît avec des aliments riches en fibres ou contenant des proportions élevées de liquides (mélasse, matières grasses) selon Olentine (1980).

\section{2 / Aspects technologiques}

\section{1 / Evaluation du comportement liant et lubrifiant}

\section{a / Méthode par compaction}

L'aptitude d'une substance à cimenter les particules peut s'évaluer indépendamment de la variabilité des conditions propres du pressage : substrat, type de filière, vapeur ajoutée, vitesse de rotation, etc. Dans le principe, le liant est ajouté à un mélange de sable et d'eau $(10 \%)$, qui est compacté sous une pression connue dans un moule cylindrique. Après extraction de la carotte ainsi formée et stabilisation à $25^{\circ} \mathrm{C}$ pendant 24 heures, la cohésion est évaluée par la résistance à l'écrasement.

Un dispositif dérivé (Le Deschault de Monredon 1990) consiste à comprimer de la même manière le mélange additif-farine entre deux poinçons (figure 2a) à une pression fixée $(200 \mathrm{MPa})$ et à enregistrer la courbe forcedéplacement. Le comportement de la farine (pouvoir lubrifiant) est caractérisé par un seuil de plasticité repéré sur la courbe et par le travail de compression (figure $2 b$ ). Le seuil de plasticité représente la pression théorique requise pour passer de l'état divisé à un état cohérent semi-continu. La masse volumique, le module d'élasticité apparent et la résistance à l'écrasement sont mesurés sur la carotte obtenue (pouvoir liant). La valeur prédictive des critères mesurés à l'aide de cette méthode a été validée par comparaison avec le pressage à échelle pilote (Le Deschault de Monredon et al 1993). L'énergie spécifique absorbée par la presse est corrélée au travail de compression, sauf avec des aliments riches en fibres qui sont plus élastiques, et la dureté des granulés avec la résistance à l'écrasement des carottes. Un exemple de relation sur des aliments contenant différents taux de sépiolite et de matière grasse est donné dans la figure 3 .

\section{Les lignosulfites sont les adjuvants d'origine organique les plus utilisés. Ils contiennent notamment de la lignine sulfonée. La présence d'un ion sulfonate en surface de cette molécule peut expliquer ses propriétés hydrophiles et tensio-actives.}


Figure 2. Courbes (a) de compression d'un échantillon d'aliment en farine (évaluation de l'effet lubrifiant) et (b) d'écrasement de la carotte formée durant la compression (évaluation de l'effet liant).

Force

(a)

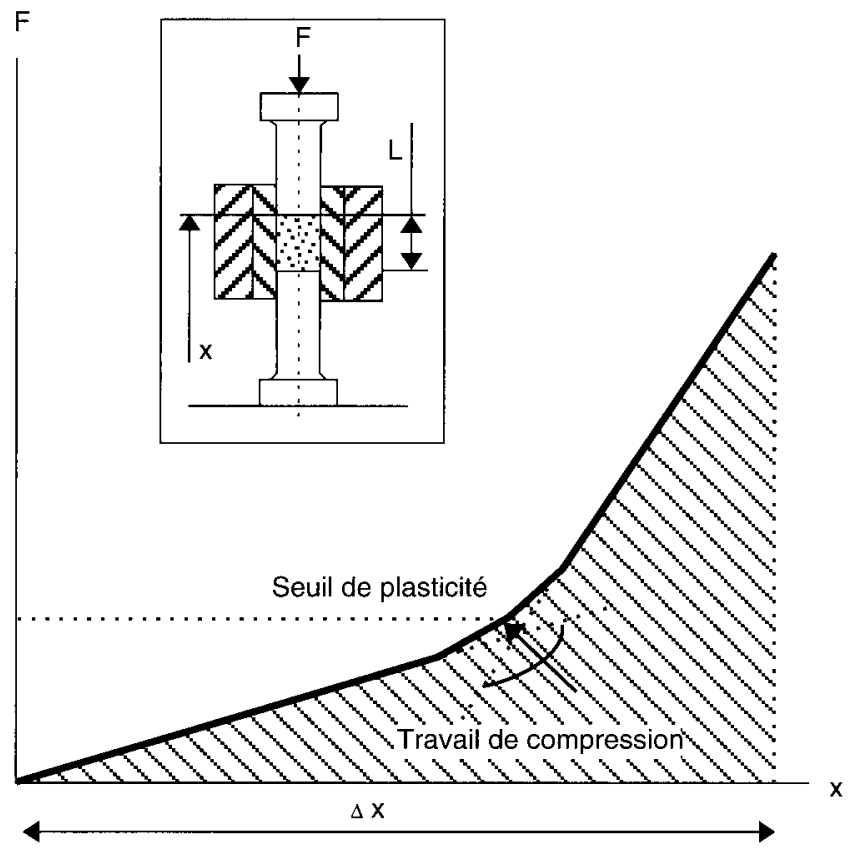

Déplacement

Force

(b)

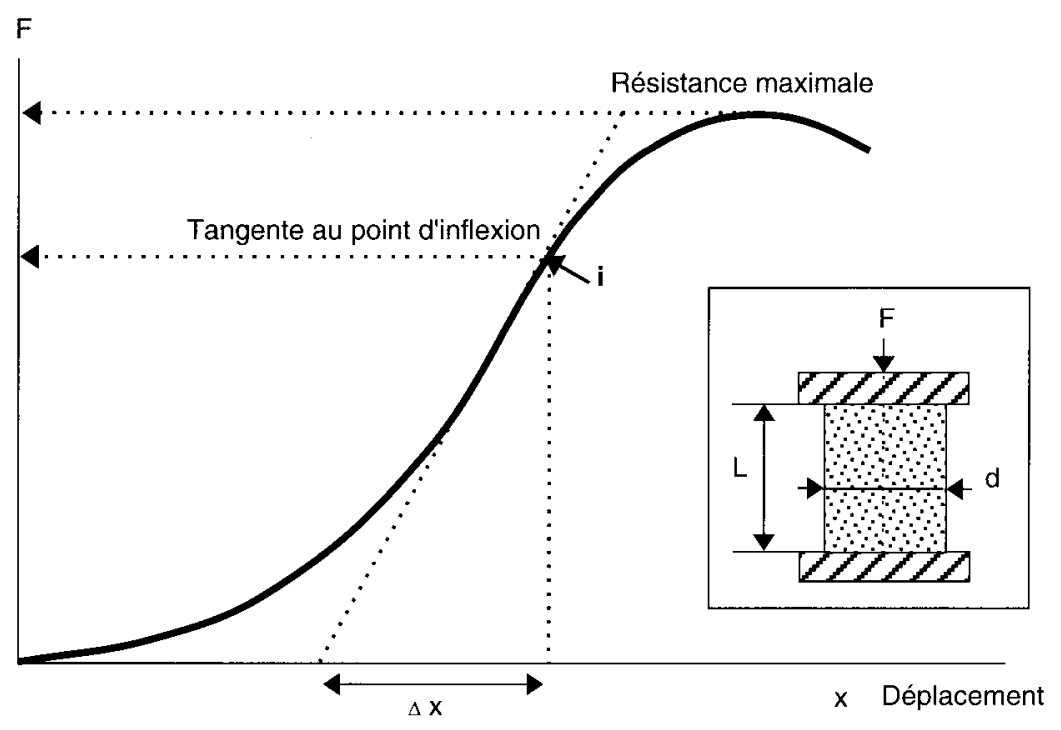

\section{b / Méthode par extrusion}

Un dispositif de principe différent a été construit (Melcion 1974) et adapté à l'étude de l'efficacité des liants (Melcion et DelortLaval 1977). Entre les deux plateaux d'une presse hydraulique de laboratoire, un dispositif d'extrusion a été mis en place. Il se compose d'un cylindre percé à sa partie inférieure d'un orifice de dimensions données (la « filière »). A travers cet orifice s'écoule la poudre comprimée par un piston mobile dans l'axe du cylindre. Un manchon chauffant thermorégulé peut maintenir le cylindre à une tem- pérature fixée. Le déplacement du piston ainsi que la force appliquée sont enregistrés par l'intermédiaire de capteurs et servent à définir un travail spécifique d'extrusion (pouvoir lubrifiant) représenté par l'aire sous la courbe force-déplacement. La farine peut être traitée à la vapeur ou non. La dureté des granulés obtenus à la sortie de la filière, identiques d'aspect à ceux élaborés à l'aide d'une presse classique, peut être mesurée à l'issue d'une période de stabilisation à température et hygrométrie contrôlée (pouvoir liant).

A titre d'exemple, ce test a permis de mettre en évidence un effet lubrifiant du lignosulfite et de la kaolinite dans des matières premières pures riches en amidon telles que l'orge, le maiis ou le son. Cet effet est réduit avec des matières premières cellulosiques comme la farine de luzerne et peut être négatif avec des tourteaux (soja), en particulier avec la kaolinite (figure 4). L'effet lubrifiant est rapidement tamponné en présence d'eau ajoutée au mélange.

\section{2 / Evaluation à échelle pilote et industrielle}

\section{a / Précautions méthodologiques}

Les lubrifiants sont utilisés dans le but de réduire la consommation d'énergie et d'accroître, si possible, le débit horaire. L'opérateur doit mesurer ces deux critères avec la meilleure précision possible. En particulier, l'intensité électrique absorbée par la presse doit être enregistrée afin d'obtenir des informations supplémentaires sur la régularité du fonctionnement de la machine durant l'essai. De plus, il est nécessaire de prendre en compte les particules fines séparées par tamisage à la sortie du refroidisseur et habituellement recyclées sur la presse. Bien des informations disponibles sont rendues inutilisables (ou partiales) en raison de l'absence de certains de ces critères.

Les liants sont utilisés dans le but d'accroître la cohésion du granulé, c'est-à-dire sa dureté et sa durabilité. La durabilité semble être le principal facteur à mesurer : un industriel souhaite obtenir des granulés qui ne se brisent pas lors des manutentions, du transport et de la distribution à la ferme. La méthode la plus connue est celle dite des "caissons tournants" qui fait l'objet d'une norme américaine ASAE S358 (Pfost et Allen 1962), ainsi que la méthode pneumatique développée par la firme suédoise Holmen (Payne 1979). Les termes durabilité ou friabilité peuvent être utilisés indifféremment : la friabilité, qui est le pourcentage de particules arrachées à l'aliment après abrasion est égale à (100 - durabilité). Il faut cependant prêter attention à la manière d'exprimer les résultats qui peut conduire à une interprétation parfois " optimiste" de l'addition d'un liant : une réduction de friabilité de 10 à $5 \%$ équivaut à un accroissement de durabilité de 90 à $95 \%$. Il s'ensuit que l'efficacité relative obtenue 
Figure 3. Relation entre tests de laboratoire et essais sur presse pilote (aliment avec 3 et $6 \%$ de suif, en présence de 2 et $4 \%$ de sépiolite).

\section{a- Travail de compression et énergie consommée par la presse}

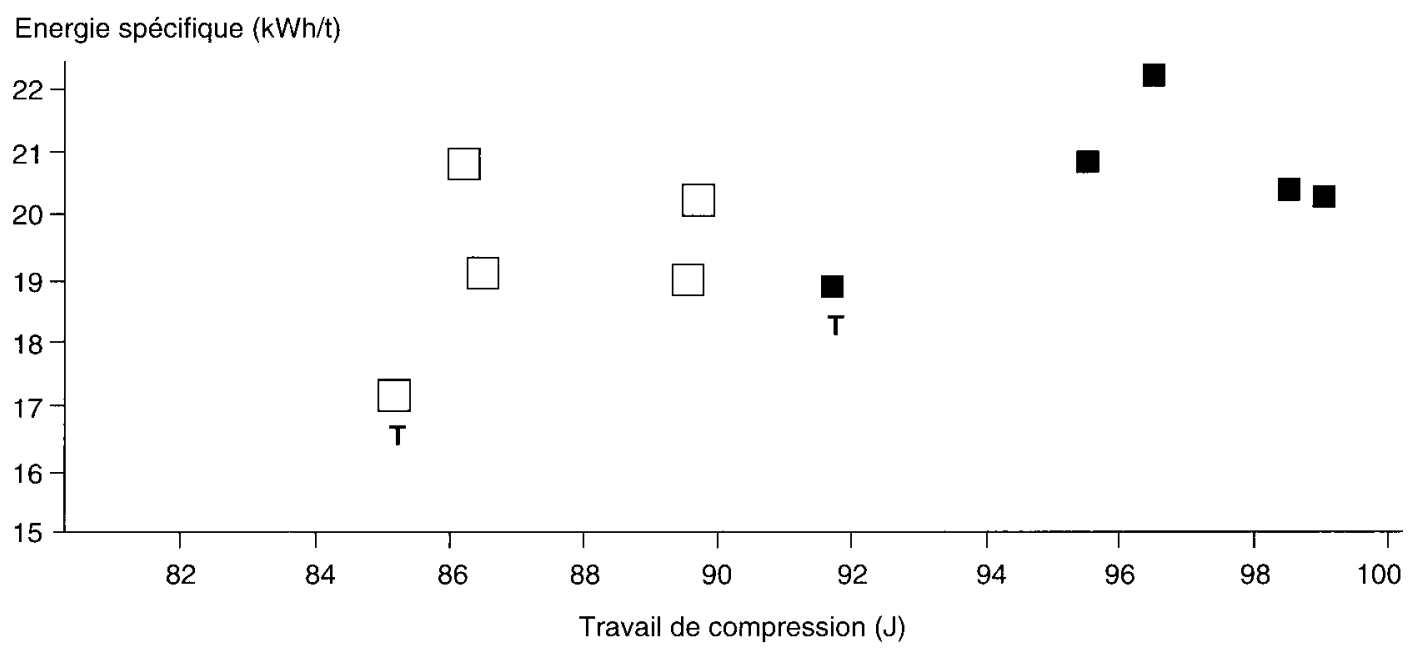

b- Résistance à l'écrasement de la carotte et dureté des granulés

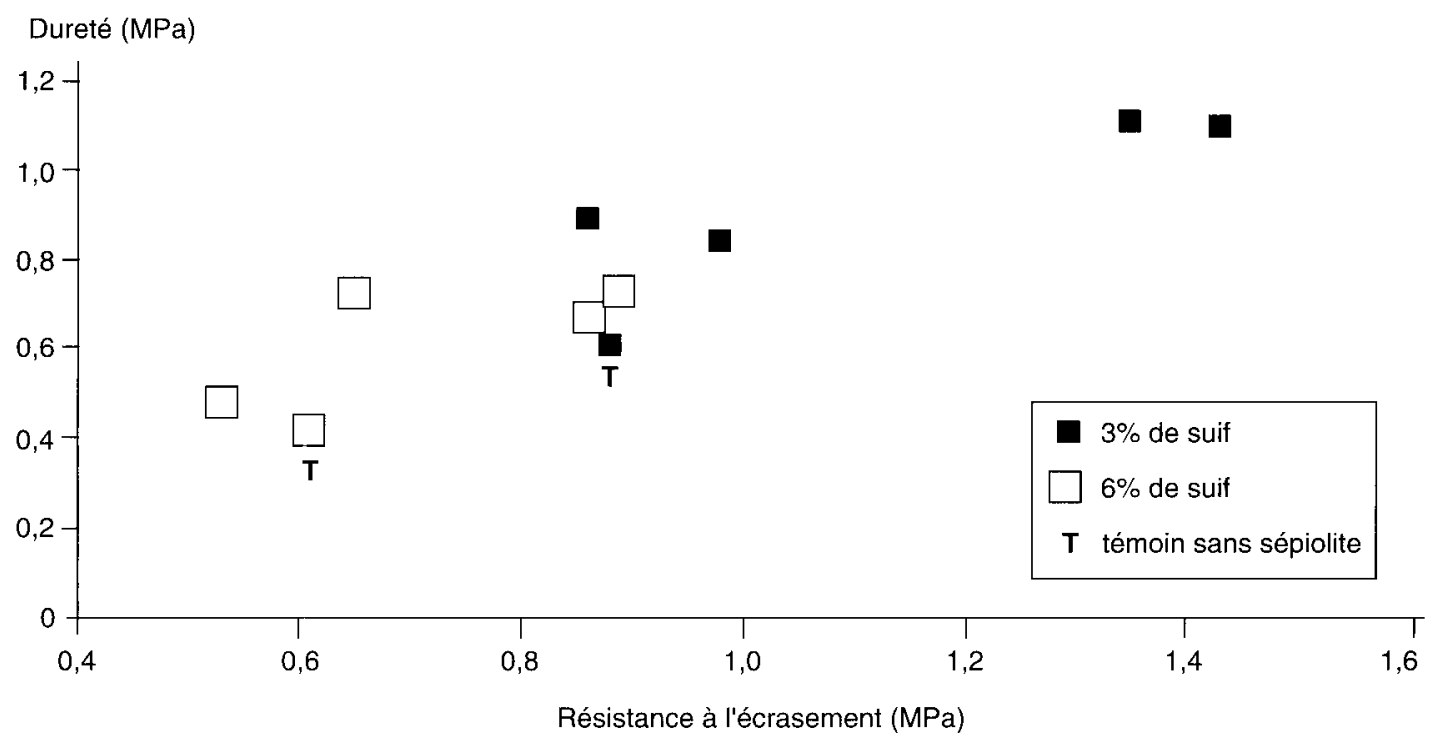

Figure 4. Evaluation de l'effet lubrifiant d'adjuvants de pressage (test d'extrusion de laboratoire).

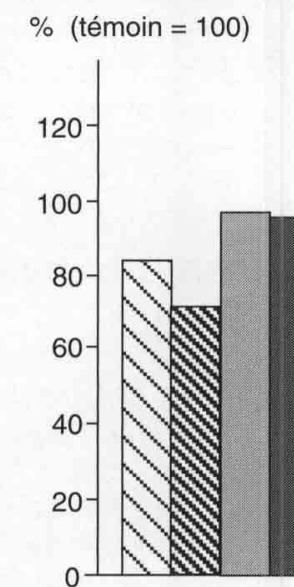

Maïs
\. Lignosulfite $2 \%$

Lignosulfite $5 \%$

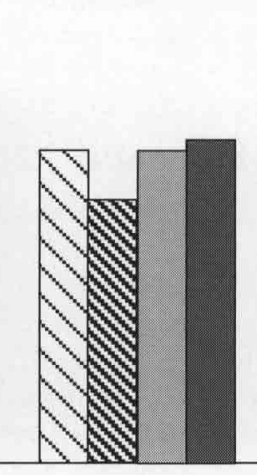

Orge
Kaolinite 2\%

Kaolinite 5\%

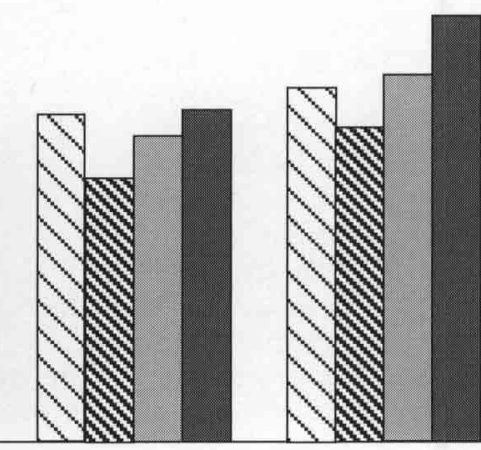

Luzerne

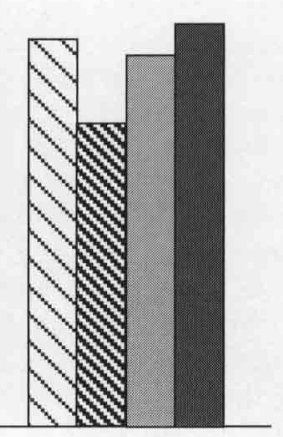

Soja
Le lignosulfite et la kaolinite ont un effet lubrifiant surtout dans les matières premières riches en amidon comme l'orge ou le maïs. 
est de $50 \%$ dans le premier cas et seulement de $5,6 \%$ dans le second. La dureté, évaluée par la résistance à l'écrasement des granulés (Delort-Laval et Drevet 1970) a une connotation quelque peu différente et se relie davantage au comportement alimentaire de l'animal (durée de repas ou de mastication, prise d'aliment).

La production de granulés est connue pour dépendre de nombreux paramètres : débit d'alimentation de la presse, quantité de vapeur incorporée, type de matière première, géométrie et usure de la filière. En raison des interrelations entre ces paramètres, il semble nécessaire de procéder à une comparaison avec ou sans liants dans les mêmes conditions de fonctionnement dans un premier temps, avant d'optimiser les résultats pour une substance donnée dans un second temps. Il est connu par exemple qu'un débit d'alimentation plus élevé, donc un temps de séjour plus faible dans la filière, conduit à une détérioration de la qualité des granulés : l'influence propre du débit peut alors interférer avec celle du liant que l'on souhaite mettre en évidence. Les performances du matériel aux plans technique et économique (production spécifique et débit horaire) seraient à établir sur la base d'une durabilité de référence, $96 \%$ par exemple selon Friedrich (1983). De plus chaque test en atelier pilote ou en usine devrait être effectué en double ou en triple.

\section{b / Tendances technologiques}

\section{- essais au stade pilote :}

Les études complètes évaluant l'efficacité technologique des liants ne sont pas nombreuses. La plupart des auteurs reconnaissent cependant aux lignosulfites une influence favorable lorsque ceux-ci sont ajoutés à des mélanges riches en matières premières amylacées, à la fois sur la durabilité et sur la consommation d'énergie, avec une interaction positive entre le taux de vapeur incorporée et le taux de liant (Friedrich et Robohm 1970, Payne 1979) surtout en ce qui concerne l'énergie spécifique. Cette influence disparaît en présence de sucres ou de lait en poudre dans les aliments pour porcelets (Bruggeman et al 1964), ou si la proportion de tourteau de soja par rapport aux céréales et au manioc (Pfost 1964) croît. Leur effet en présence de matière grasse est plus incertain. Dans les essais conduits à débit constant, et pour des taux d'incorporation de 1 à $2 \%$, la réduction d'énergie spécifique est plus faible, inférieure à $10 \%$, et souvent masquée par l'addition d'eau ou de vapeur. Le lignosulfite s'avère souvent être davantage un liant qu'un lubrifiant. La kaolinite et une carboxy-méthyl-cellulose dans un aliment pour lapin plus riche en fibres, n'ont pas entraîné d'effet pratique significatif sur la consommation d'énergie spécifique. Exprimée en termes de friabilité, la cohésion de l'aliment a été cependant réduite de façon plus marquée (Melcion et
Delort-Laval 1977). D'autre part, il n'y a généralement pas de proportionnalité apparente entre le pourcentage de liant ajouté, quel qu'il soit, et le résultat technologique.

En vue d'éviter la variabilité due à la complexité des mélanges, kaolinite et lignosulfite ont été ajoutés à des matières premières pures agglomérées à sec (sans vapeur). Par rapport aux témoins sans adjuvants, la consommation d'énergie est réduite avec les céréales et le son de blé, identique avec la farine de luzerne et accrue avec le tourteau de soja quels que soient les adjuvants testés. La friabilité des granulés est réduite, davantage avec le lignosulfite qu'avec la kaolinite, sauf pour les matières premières (luzerne, soja) dont les produits sont déjà très peu friables (Melcion et Delort-Laval 1977).

En raison de leur capacité d'absorption élevée des liquides, certaines argiles (bentonite, sépiolite) peuvent être utilisées comme support d'incorporation des matières grasses en limitant la perte de cohésion du granulé (Lopez et Alvarez 1991). A débit d'alimentation constant sur des aliments pour volaille contenant 3 ou $6 \%$ de matière grasse (suif), on note, sur la dureté, un effet significatif de l'addition de 2 ou $4 \%$ de sépiolite $(+28$ et $+74 \%$ en moyenne respectivement en valeur relative par rapport aux témoins dont la cohésion est bien connue pour être médiocre) (figure 3 déjà citée). Par contre, la durabilité des aliments est peu modifiée. Le prémélange de la graisse à l'argile avant de l'ajouter à l'aliment n'apporte pas d'avantage supplémentaire.

D’une façon générale, les résultats obtenus sont encore fragmentaires et demandent d'autres recherches.

\section{- essais au stade industriel :}

A l'échelle industrielle, avec différents types d'aliments, plus le niveau d'énergie consommée est bas (la production spécifique de la presse exprimée en $\mathrm{kg} / \mathrm{kWh}$ est plus élevée), et plus l'efficacité relative de l'addition d'adjuvants paraît faible. C'est le cas avec l'aluminate de calcium (Nivet 1993, tableau 4).

D'un autre coté, les différences constatées en milieu industriel sont souvent limitées (tableau 5) et peuvent être masquées par des événements aléatoires difficiles à éviter pendant les essais en usine.

\section{3 / Efficacité zootechnique des adjuvants de pressage}

Les adjuvants minéraux ne possèdent aucune valeur alimentaire par eux-mêmes. Les lignosulfites, qui contiennent 11 à $35 \%$ de glucides, peuvent avoir une certaine valeur énergétique. La variabilité de la réponse animale décrite dans la littérature est reliée au type d'animal, à la composition 
Tableau 4. Intérêt relatif d'un liant en fonction du rendement de la presse et de la manière d'exprimer les résultats (essais industriels Tecaliman : Nivet 1993).

\begin{tabular}{|c|c|c|c|c|}
\hline Type d'aliment & Critères & Témoin & $\begin{array}{c}0,6 \% \text { d'aluminate } \\
\text { de calcium }\end{array}$ & $\begin{array}{c}\text { Variation } \\
(\%)\end{array}$ \\
\hline $\begin{array}{l}\text { Poulets chair } \\
\text { (9\% graisse) }\end{array}$ & $\begin{array}{c}\text { prod. spécifique } \\
(\mathrm{kg} / \mathrm{kWh}) \\
\text { friabilité }(\%) \\
\text { durabilité }(\%)\end{array}$ & $\begin{array}{l}145 \\
13,4 \\
86,6\end{array}$ & $\begin{array}{r}157,5 \\
10,3 \\
89,7\end{array}$ & $\begin{array}{r}8,6 \\
-31 \\
3,6\end{array}$ \\
\hline Bovins & $\begin{array}{c}\text { prod. spécifique } \\
\text { (kg/kWh) } \\
\text { friabilité }(\%) \\
\text { durabilité }(\%)\end{array}$ & $\begin{array}{r}92 \\
3,6 \\
96,4\end{array}$ & $\begin{array}{r}92,5 \\
\\
2,3 \\
97,7\end{array}$ & $\begin{array}{r}0,5 \\
-60 \\
1,3\end{array}$ \\
\hline Porcs & $\begin{array}{c}\text { prod. spécifique } \\
\text { (kg/kWh) } \\
\text { friabilité }(\%) \\
\text { durabilité }(\%)\end{array}$ & $\begin{array}{r}88,7 \\
\\
4,3 \\
95,7 \\
\end{array}$ & $\begin{array}{r}104 \\
\\
3,5 \\
96,5 \\
\end{array}$ & $\begin{array}{r}17,2 \\
-25 \\
0,9\end{array}$ \\
\hline Lapins & $\begin{array}{c}\text { prod. spécifique } \\
\text { (kg/kWh) } \\
\text { friabilité }(\%) \\
\text { durabilité }(\%)\end{array}$ & $\begin{array}{r}42,9 \\
\\
1,3 \\
98,7\end{array}$ & $\begin{array}{r}43,2 \\
\\
0,9 \\
99,1\end{array}$ & $\begin{array}{c}0,7 \\
-42 \\
0,4\end{array}$ \\
\hline
\end{tabular}

de l'aliment et au type d'adjuvant, y compris dans une même famille de produits, et à ses propriétés physiques face à la mise en ouvre technologique. Ces dernières informations sont d'ailleurs souvent absentes.

\section{1 / Chez le ruminant}

La bentonite a été utilisée chez le ruminant comme un moyen de contrôle du $\mathrm{pH}$ dans le rumen, comme source de minéraux, ou comme agent liant. Un mélange (en milieu humide) de bentonite de sodium et de tourteau de soja accroît l'utilisation de l'azote chez l'agneau (Britton et al 1978). Cet effet bénéfique a été attribué à des interactions d'adsorption entre les protozoaires cilies du rumen ou leur substrat de croissance, et la bentonite. A l'aide d'une technique de simulation in vitro du rumen (Rusitec), Wallace et Newbold (1991) ont mis en évidence une réduction du nombre de protozoaires accompagnée d'une baisse de la production d'ammoniac, attribuée à une sorte d'enrobage des protozoaires ciliés. La bentonite apparaîtrait finalement comme un moyen de réduire partiellement l'activité de la micro-faune, conduisant ainsi à une augmentation du flux de protéines sortant du rumen.

Les lignosulfites sont utilisés depuis longtemps dans les aliments du ruminant, soit comme liants, soit comme additifs dans les compléments liquides. La vache laitière demande un certain délai d'accoutumance ( 3 jours) à une ration contenant $2 \%$ de lignosulfite, mais en aucun cas il n'a été observé une diminution des quantités de matière sèche ingérées. Les essais in vitro menés au Grassland Research Institute (1968) suggèrent que les sucres du lignosulfite de calcium sont fermentés pour produire des acides gras volatils avec très peu d'influence sur la digestibilité des fourrages lorsque le lignosulfite est ajouté à $2,5 \%$. A taux plus élevés (2 à $8 \%$ MS), le lignosulfite d'ammonium devient une source d'azote non protéique pour le mouton et le bovin à l'engrais. La valeur alimentaire

Tableau 5. Efficacité du lignosulfite incorporé dans 2 types d'aliments (essais industriels Tecaliman : Nivet 1993).

\begin{tabular}{|l|c|c|c|}
\hline \multicolumn{1}{|c|}{ Aliment } & Critères retenus & Témoin & Lignosulfite 1,5 \% \\
\hline pour & débit réel $(\mathrm{t} / \mathrm{h})$ & 7,8 & 9 \\
poulet de chair & prod. spécifique $(\mathrm{kg} / \mathrm{k}$ Wh) & 70,8 & 83,1 \\
& taux de fines recyclées & & \\
& sur la presse $(\%)$ & 4,4 & 2,3 \\
& durabilité $(\%)$ & 89,7 & 88,7 \\
& dureté $\left(\mathrm{MPa}^{*}\right)$ & 0,89 & 0,92 \\
\hline pour lapin & débit réel $(\mathrm{t} / \mathrm{h})$ & 2,47 & 2,58 \\
& prod. spécifique $(\mathrm{kg} / \mathrm{kWh})$ & 23,3 & 24,4 \\
& taux de fines recyclées & 1 & 1 \\
& sur la presse $(\%)$ & 98,6 & 98,6 \\
& durabilité $(\%)$ & 2,3 & 2,6 \\
\hline
\end{tabular}

* MPa $=$ Mégapascals $\left(1 \mathrm{MPa}=10,2 \mathrm{~kg} / \mathrm{cm}^{2}\right)$.

\section{L'addition de} lignosulfite, à un taux $<2 \%$, aux rations des ruminants a très peu d'effet sur la digestibilité des fourrages. Au-delà de $2 \%$, le lignosulfite d'ammonium devient une source d'azote non protéique. 
de l'azote provenant de l'ion ammonium associé est semblable à celle de l'urée (Croyle et al 1975), mais l'efficacité alimentaire semble légèrement réduite au-delà du taux d'incorporation de $4 \%$ de la matière sèche (Chang et al 1977).

Le traitement de tourteau de soja ou de colza par le lignosulfite (5-10\%) à température élevée $\left(90-95^{\circ} \mathrm{C}\right)$ met à profit la réaction entre les groupements sulfonates du lignosulfite et les groupements aminés des protéines. Il entraîne in situ un effet de tannage et une réduction de leur dégradabilité (McAllister et al 1993).

\section{2 / Chez le porc}

D'après Collings et al (1980), la bentonite de sodium $(2,5 \%)$ tend à accroître l'efficacité alimentaire pendant la période de démarrage, mais pas en période de croissance ou de finition. Elle n'a aucune incidence sur la qualité de la carcasse. Un effet de substitution semble alors s'exercer entre la bentonite et un antibiotique (la lincomycine) en termes de niveau de consommation et de gain de poids. Ces résultats sont différents de ceux de Rivera et al (1978) avec la kaolinite. L'adjonction de 1 à $3 \%$ de kaolinite dans la ration du porcelet ne modifie en rien la vitesse de croissance ou l'indice de consommation. Dans d'autres essais rapportés par Dunoyer (1986), seule la vitesse de croissance est légèrement améliorée, peutêtre en raison d'une réduction des diarrhées.

Ces effets ne sont pas toujours bien compris. L'addition d'argile équivaut à une dilution de l'aliment conduisant théoriquement à des performances réduites si l'on exprime les résultats par rapport à la ration totale. Plusieurs hypothèses sur les mécanismes d'action de ces substances ont été proposées. Avec la sépiolite, l'hypothèse la mieux acceptée serait un ralentissement du transit intestinal dû à la modification de la viscosité du contenu grâce à la capacité d'absorption d'eau de l'argile. L'action des enzymes intestinaux serait prolongée, accroissant ainsi l'efficacité de la digestion et l'absorption des nutriments (Lopez et Alvarez 1991). Une autre explication pourrait être une interaction entre la dureté et/ou l'élévation de température des granulés quittant la filière, liée à la présence de l'adjuvant, et la consommation par l'ani- mal. Une dureté excessive tend à réduire le niveau de consommation, des températures plus élevées accroissent la digestibilité de l'énergie de rations contenant de l'amidon de céréales ou de légumineuses. Des interactions avec la microflore intestinale peuvent également être invoquées.

Chez le porcelet, l'accroissement de durabilité reliée à l'addition de sépiolite ( $2 \%$ en substitution au maîs) semble conduire à une réduction de la consommation alimentaire mais aussi de la croissance avec des rations peu énergétiques (Castaing 1989). Lindice de consommation est identique dans ce cas à celui du témoin, mais meilleur avec des rations à plus haute teneur en énergie (tableau 6). Dans les deux cas, l'indice de conversion de l'énergie est accru (2,8 et $4,5 \%$ en valeur relative respectivement) avec l'argile. Chez le porc en croissance ou en finition (20-100 et même $150 \mathrm{~kg}$ de poids vif), les performances ne diffèrent pas significativement entre groupes expérimentaux et groupes témoins, montrant par là un effet de compensation de la dilution de la ration avec le liant (Parisini et al 1993). Si mâles et femelles sont étudiés séparément (Castaing 1989), l'incorporation du liant a un léger effet dépressif $(2,5$ à $3 \%$ ) chez les mâles castrés, de même ordre de grandeur que celui de la dilution de l'aliment, mais non significatif chez la femelle. L'indice de conversion de l'énergie est alors plus élevé. Une couleur plus claire de la viande avec les animaux (103 kg) recevant de la sépiolite a été notée (Parisini et al 1993), ainsi qu'une tendance à un dépôt plus important de lard chez le porc lourd italien (150 kg).

\section{3 / Chez la volaille}

Linfluence des lignosulfites a été particulièrement étudiée chez la volaille. Les aliments pour poulet de chair contiennent en effet des proportions élevées de matières grasses qui nécessitent parfois de recourir à des adjuvants de pressage.

D'une manière générale, en présence de $3 \%$ de lignosulfite de calcium, la digestibilité des composants majeurs de l'aliment tend à décrô̂tre avec l'âge de l'animal (Kivimäe 1978). Parallèlement, la consistance des fientes est plus "relâchée » et leur teneur en

Tableau 6. Effets zootechniques de l'incorporation de sépiolite dans 2 aliments pour porcelet (Castaing 1989).

\begin{tabular}{|c|c|c|c|c|c|}
\hline Aliment & sans & avec & sans & avec & $\begin{array}{c}\text { Effet } \\
\text { de l'argile }\end{array}$ \\
\hline Niveau énergétique (kcal) & 3300 & 3300 & 3100 & 3100 & \\
\hline Teneur en sépiolite $(\%)$ & 0 & 2 & 0 & 2 & \\
\hline Température des granulés $\left({ }^{\circ} \mathrm{C}\right)$ & 61 & 67 & 62 & 65 & non \\
\hline Friabilité ASAE (\%) & 21,4 & 11,3 & 12 & 6,9 & analysé \\
\hline Consommation $(\mathrm{kg} / \mathrm{j})$ & 0,945 & 0,942 & 0,96 & 0,939 & 0,09 \\
\hline Vitesse de croissance $(\mathrm{g} / \mathrm{j})$ & 522 & 533 & 524 & 514 & $\mathrm{~ns}$ \\
\hline Indice de consommation & 1,81 & 1,77 & 1,84 & 1,83 & 0,21 \\
\hline Indice énergétique (Mcal/kg) & 5,95 & 5,68 & 5,69 & 5,53 & \\
\hline
\end{tabular}


eau augmente (de 61,5 à 73,5\%). L'accroissement de teneur en eau est attribué à la teneur du lignosulfite en composés soufrés qui agiraient comme des irritants de la muqueuse du tube digestif. L'effet laxatif est moindre avec 1 à $2 \%$ de lignosulfite dans l'aliment. A $5 \%$, les animaux montrent des caeca distendus et des contenus caecaux brun foncé, luisants, à la fois "tenaces " et gélatineux jusqu'à l'âge de l'abattage. Cette distension des caeca tend à accroître le risque de rupture au moment de l'éviscération lors de l'abattage. A six semaines, les villosités intestinales sont aplaties (Proudfoot et de Witt 1976). Avec le lignosulfite de calcium provenant de résineux (épicéa), la teneur en eau des fientes est supérieure à celui provenant de feuillus (hêtre) : elle est de 71,0 et $72,5 \%$ respectivement contre $68,7 \%$ avec le témoin. La consommation d'eau est accrue parallèlement (Leibetseder et Skalicky 1974). Une accentuation de ces effets est observée chez les poulets contaminés par la coccidiose.

Entre 0,5 et $2,5 \%$ de lignosulfite dans la ration, ni l'indice de consommation, ni la vitesse de croissance des animaux ne sont modifiés significativement par rapport au témoin (Waldroup et al 1982). Il n'existe pas non plus de différence dans le rendement en viande et le classement des carcasses. Audessus de 2,5 \%, l'usage du liant entraîne une baisse limitée des performances, et un accroissement possible de la mortalité. Il existe une liaison significative entre le taux de lignosulfite dans la ration et le degré de salissure du plancher de la cage et du plumage lié à la dispersion accrue des gouttelettes lors de l'abreuvement.
L'absence constatée d'effets négatifs par rapport au témoin de la bentonite de sodium $(2,5 \%)$ sur la croissance et l'efficacité alimentaire chez le poulet de chair rejoint les conclusions des essais menés sur le porc. Tout se passe comme si l'effet de dilution dû à la présence d'argile était compensé par une meilleure utilisation digestive des composés alimentaires, peut-être grâce à un ralentissement du bol alimentaire dans l'intestin (Salmon 1985). La teneur en eau des fientes $(65,6 \%)$ est réduite significativement par rapport au témoin $(68,7 \%)$ avec $2 \%$ de bentonite de sodium dans la ration (Leibetseder et Skalicky 1974).

Plus récemment, l'incorporation de 1,5\% de sépiolite en substitution au maïs a été étudiée chez le poulet de chair dans un aliment en farine en vue de vérifier l'hypothèse de l'accroissement du temps de transit (Tortuero Cosialls et al 1992). L'incorporation d'argile dans la ration se traduit par un allongement de la durée de transit $(2-3 \mathrm{~h}$ pour $87,5 \%$ des oiseaux avec l'aliment contenant $1,5 \%$ de sépiolite contre $1,5-1,75 \mathrm{~h}$ pour $62,5 \%$ des oiseaux avec l'aliment témoin). Aucune différence significative de croissance et d'efficacité alimentaire n'a été mise en évidence sur la totalité de la période 10-42 jours, mais de légères différences apparaissent selon l'âge, positives dans le jeune âge, négatives plus tard, le besoin en énergie augmentant avec l'âge. La disponibilité de l'énergie pourrait être influencée par la vitesse de transit.

L'influence des liants peut être déterminée à travers leur efficacité relative sur l'énergie métabolisable apparente (EMA) de la ration. L'EMA d'aliments contenant de la kaolinite

L'influence de
l'adjuvant sur
l'EM apparente de
l'aliment est
variable selon sa
nature mais
ne dépend pas
de son taux
d'incorporation.

Figure 5. Influence de l'incorporation d'adjuvants de pressage sur l'énergie métabolisable apparente corrigée d'un aliment pour poule pondeuse (Melcion et Delort-Laval 1977).

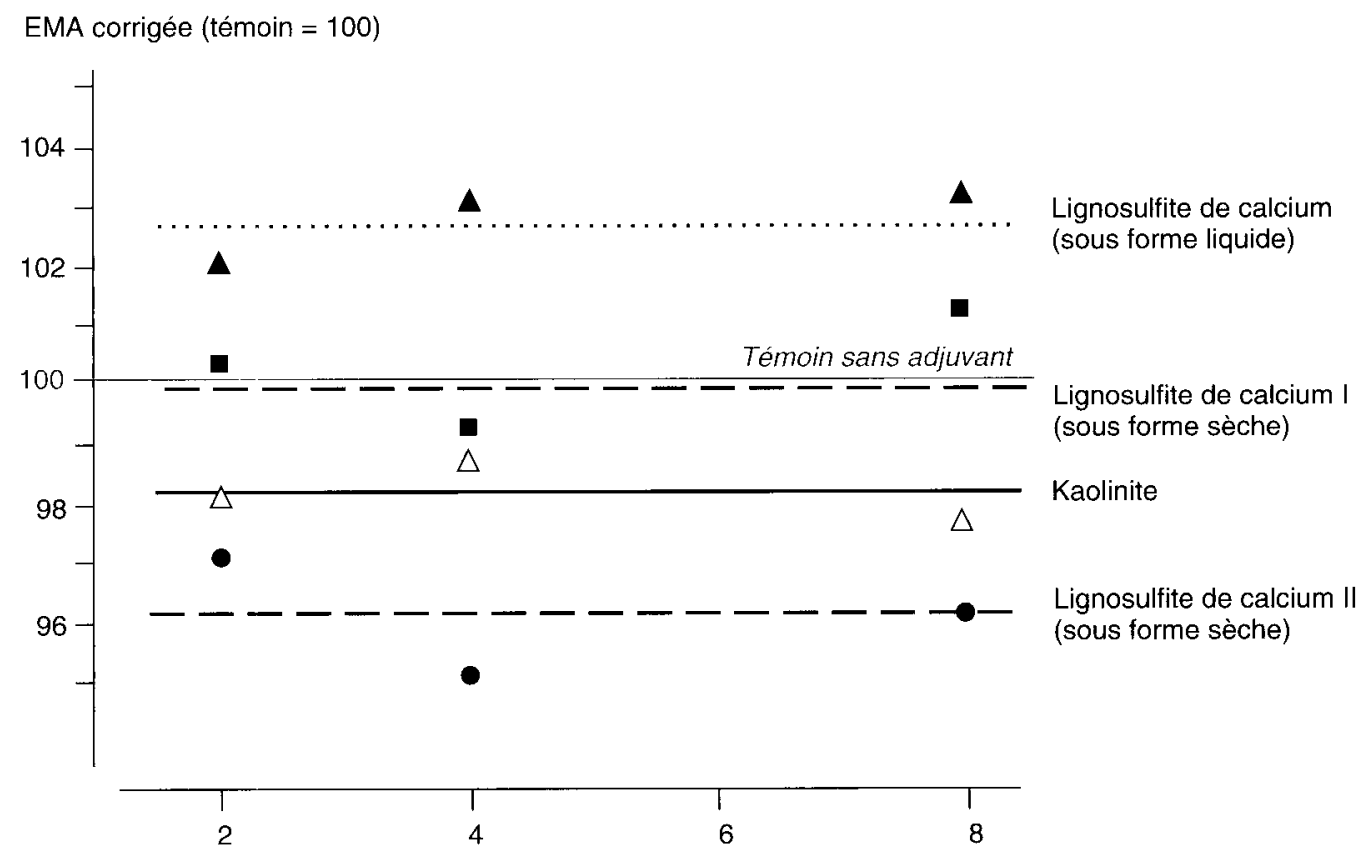

Taux d'incorporation de l'additif (\%) 
et des lignosulfites (sous forme liquide ou solide) a été corrigée en tenant compte de la dilution apportée par le liant. L'influence du liant est positive ou négative (Melcion et Delort-Laval 1977) mais toujours indépendante de son taux d'incorporation (figure 5). Dans le cas le plus favorable (lignosulfite de calcium liquide), l'accroissement d'EMA est de $75 \mathrm{kcal} / \mathrm{kg}$, soit environ $2,5 \%$ de la valeur du régime. Le liant n'aurait pas de valeur énergétique propre, et une valeur constante devrait le cas échéant lui être attribuée.

\section{Conclusions}

Un adjuvant de pressage doit être adapté à l'aliment (teneur en matière grasse), et aux conditions de pressage (épaisseur de filière, eau, vapeur ajoutée) en fonction de ses propriétés spécifiques. D'un autre coté, lorsqu'on choisit d'utiliser un adjuvant, il est nécessaire d'évaluer son taux d'incorporation, son coût, notamment de stockage (prise en masse !) et de manutention, ainsi que l'avantage technique ou commercial apporté.

Des dispositifs expérimentaux et des méthodes sont disponibles pour tester l'efficacité de ces substances après incorporation dans un aliment; les résultats obtenus en laboratoire sont corrélés avec les essais à échelle pilote. Il convient cependant d'être prudent quant à l'extrapolation à la pratique industrielle. Cela signifie un contrôle précis des performances de la presse, la mesure des caractéristiques physiques des aliments agglomérés ainsi que l'emploi de méthodologies expérimentales adaptées.

Les essais montrent que les adjuvants de pressage sont souvent plus efficaces comme liants que comme lubrifiants. La réponse est plus favorable avec des aliments contenant des céréales, dont l'agglomération est aisée, qu'avec des aliments à teneur élevée en fibres ou en protéines.

Leur emploi ne se justifie évidemment pas si l'équipement est bien maîtrisé et que l'on produit déjà des aliments agglomérés de bonne qualité. D'un autre côté, les conditions de pressage sont rarement idéales, et les résultats peuvent être aléatoires. L'emploi d'adjuvants peut alors se justifier ponctuellement pour éviter d'avoir à changer une filière, ou pour compenser une médiocre aptitude au pressage d'un aliment donné.

Leurs effets nutritionnels sont difficiles à évaluer, d'autant que les taux d'incorporation sont bas, et encore mal élucidés. Ils n'ont pas de valeur nutritionnelle directe, mais peuvent avoir une valeur indirecte par l'accroissement de la densité des granulés et de leur dureté (lignosulfites) susceptible de limiter le gaspillage de l'aliment et/ou par leur effet sur la pression osmotique à l'intérieur du tube digestif et la modification potentielle de transit intestinal qu'il peuvent induire (argiles). Les liants, à l'origine utilisés comme additifs technologiques, pourraient dans certains cas être considérés comme additifs à vocation nutritionnelle.

Leur incorporation dans un aliment peut entraîner un effet positif ou négatif sur l'énergie métabolisable du reste de la ration pour le poulet de chair, dont il a été démontré qu'il était indépendant du taux d'incorporation dans la gamme testée. D'autres recherches de physiologie digestive sur les effets associatifs au cours de la digestion des composants dans un aliment seraient nécessaires pour compléter nos connaissances sur l'efficacité biologique de ces substances dans les aliments agglomérés.

Certains des résultats présentés dans cet article ont été obtenus grâce à l'aide de la société TOLSA (Madrid), que nous remercions vivement.

\section{Références bibliographiques}

Alvarez A., 1984. Sepiolite : properties and uses. In : Palygorskite-sepiolite occurences, genesis and uses. A. Singer \& E. Galan (eds), Elsevier, Amsterdam (NL), 253-287.

Britton R.A., Colling D.P., Klopfenstein T.J., 1978. Effect of complexing sodium bentonite with soybean meal or urea in vitro ammonia release and nitrogen utilization in ruminants. J. Anim. Sci., 46, 17381747.

Bruggeman J., Niesar K.H., Walter U., 1964. Einfluss von Presshilfstoffen auf Pressleistung und Pellethärte im grosstechnischen Versuch. Kraftfutter, 47, 414-420.

Caillère S., Hénin S., Rautureau M., 1982. Minéralogie des argiles. $1:$ Structure et propriétés physicochimiques. INRA n 8 ( $2^{\circ}$ éd.), Masson, Paris, $184 \mathrm{p}$.

Castaing J., 1989. Effet de l'introduction de $2 \%$ de sepiolite "Exal " dans les aliments à deux niveaux énergétiques présentés en granulés pour porcelet et porcs charcutiers. J. Rech. Porcine en France, 21, $51-58$.

Chan S.L.H., Baker C.G.J., Beeckmans J.M., 1976. Floculating properties of high molecular weight lignosulfonate. Powder Technol., 13, 223-230.

Chang F.S., Dyer I.A., Johnson R.J., 1977. Performances of feedlot cattle and rumen microorganisms as influenced by lignosulfonate. J. Anim. Sci., 45, 878-884.

Collings G.F., Thomasson S.A., Ku P.K., Miller E.R., 1980. Sodium bentonite in swine diets. J. Anim. Sci., 50, 272-277.

Croyle R.C., Long T.A., Hershberger T.V., 1975. Evaluation of ammonium lignin sulfonate as a non-protein nitrogen source for sheep. J. Anim. Sci., 40, 1144-1149.

David J., Lefumeux J., 1973. Conduite et entretien des presses. L. David éd., La Jarrie (France), 112 p. 
Delort-Laval J., Drevet S., 1970. Méthode d’appréciation de la dureté des aliments agglomérés. Ind Alim. Anim., 213, 43-54.

Dumée G., Michelin B., 1970. Emploi des lignosulfites dans la granulation des sons. Bull. Anciens Élèves Ecole Française de Meunerie, 239, 232-237.

Dunoyer C., 1983. Intérêt des argiles en nutrition animale. Application à la diététique canine. Thèse doctorat vétérinaire E.N.V. Alfort, 163 p.

Friedrich W., 1983. Warum ist Dampfzugabe beim Pelletieren wirtschaftlicher als Wasser? Die Mühle 120 (14), 173-178.

Friedrich W., Robohm K.F., 1970. Pressversuche mit Ligninsulfonat Bindemitteln. Kraftfutter, 53, 344349 .

Gustavson K.H., 1956. The chemistry of tanning process. Academic Press, New-York, pp. 223-229.

Hogan D., 1974. Molasses additives derived from wood. N.F.I.A. Congress, Decatur, Ill, feb.

Kivimäe A., 1978. Effects of lignosulphonates on poultry when used as a binder in compounded feed. Arch. Geflügelk., 42, 238-245.

Le Deschault de Monredon F., Giboulot B., Papineau P., 1993. Axial compression into a cylindrical cell as a model for studying the pelleting of feed powders. 3) Comparison with an industrial ring die press. Sci. Aliments, 13, 663-675.

Leibetseder J., Skalicky M., 1974. Über die Beeinflussung des Wassergehaltes im Hühnerkot durch Presshilfsmittel. Arch. für Geflügelkunde, 38 (2), 57-60.

Lopez A., Alvarez A., 1991. Properties and applications of sepiolite in animal feedingstuffs. Feed Compounder, sept., 28-31.

McAllister T.A., Cheng K.J., Beauchemin K.A., Bailey D.R.C., Pickard M.D., Gilbert R.P., 1993. Use of lignosulfonate to decrease the rumen degradability of canola meal protein. Can. J. Anim. Sci., 73, 211-215.

Melcion J-P., 1974. Nouvelle méthode d'étude de l'agglomération des aliments composés. Prix Protector International, $53 \mathrm{p}$.

Melcion J-P., Delort-Laval J., 1977. Effet des liants sur la production, les caractéristiques physiques et la valeur nutritive des aliments agglomérés. Bull. Anciens Elèves Ecole Française de Meunerie, 279, 148-164.

Meyers S., 1991. Pellet binders for shrimp feeds. Feed International, 12 (3), 22-28.

Nivet H., 1993. Emploi des liants dans l'agglomération des aliments des animaux. In : La granulation des aliments composés. Bull. Spécial Tecaliman $\mathrm{n}^{\circ} 17,147-167$

Olentine C., 1980. Pelleting aids. Feed Management, 31 (11), 8-12.

Parisini P., Sardi L., Panciroli A., Coppa C., 1993. Effetti della sustituzione con sepiolite di parte de la mangime nell'alimentazione del suino pesante. Atti $10^{\circ}$ Congresso Nazional ASPA, 459-464.

Payne J.D., 1979. Recent developments in the application of lignosulphonate binders. Holmen Pelleting Symposium, London (UK), 20 nov., G1-G14.

Pfost H.B., 1964. The effect of lignin binders, die thickness and temperature on the pelleting process. Feedstuffs, 36, 20-21.

Pfost H.B., Allen R.N., 1962. A standard method of measuring pellet durability. Proc. Feed Prod. School, Kansas City, 12-14 nov, 25-29.

Proudfoot F.G., de Witt W.F., 1976. The effect of the pellet binder "Lignosol FG " on the chicken's digestive system and general performance. Poultry Sci., $55,629-631$.

Rivera E.R., Armstrong W.D., Clawson A.J., Lenerud A.C., 1978. Effect of dietary oats and kaolin on performance and incidence of diarrhea of weanling pigs. J. Anim. Sci., 46 (6), 1685-1693.

Salmon R.E., 1985. Effect of pelleting, added sodium bentonite and fat in a wheat based diet on performance and carcass characteristics of small white turkeys. Anim. Feed Sci. and Technol., 12, 223-232.

Storebakken T., 1985. Binders in fish feeds. Aquaculture, 47, 11-26.

Tortuero Cosialls F., Fernandez Gonzalez E., Martin martin L., 1992. Efectos de la sepiolita en la dieta sobre el crecimiento, las medidas viscerale y el transito intestinal en pollos. Arch. Zootec., 41, 209-217.

Valaris M., Harper W., 1973. Effect of CMC on the proteolysis of casein by immobilized pepsin. J. Food Sci., 38, 477-483.

Waldroup P.W., Ritchie S.J., Ramsey B.E., 1982. Effects of lignin sulfonate pellet binder on pellet quality and feeding value for broilers. Feedstuffs, 54 (3), 33 .

Wallace R.J., Newbold C.J., 1991. Effects of bentonite on fermentation in the rumen simulation technique (rusitec) and on rumen ciliate protozoa. J. Agric. Sci., Cambridge, 116, 163-168.

Wolter R., Dunoyer C., Henry N., Seegmuller S., 1990. Les argiles en alimentation animale : intérêt général. Rec. Médecine Vétérinaire, 166, 21-27.

\footnotetext{
Use of binders in feed pelleting : technological and nutritional aspects.

Binders (or pelleting aids) are additives which are incorporated to a feed in low concentrations $(0.5$ to $2.5 \%)$ in order to increase the durability and the hardness of pellets and the efficiency of pelleting. These substances could be of organic or mineral origin. Among the organic substances
}

Abstract

used, lignosulfonates are provided as by-products by the paper industry. Mineral substances are mainly from the clay family. Other substances, such as polysaccharides could be used in marine animals feed.

Laboratory tests may provide information on the binding and/or the lubricating properties of the pelleting aids according to their moisture level 
and type of raw material. Pilot tests for the addition of lignosulfonates have generally shown a positive influence on the mechanical properties of pellets and a tendancy to decrease the energy consumption mainly with starch-rich feeds or materials. The effect of mineral substances is quite variable and depends on the pelleting conditions. At industrial scale small differences can be buffered by hazardous variations.

Generally, binders have no nutritive value per se. Lignosulfonates and other organic compounds are exceptions as they have a low sugar content. Usually, mineral binders are considered to be diluting components of the diet. An indirect effect on the feed conversion ratio could be rela- ted to the fact that the pellets are harder. Attention has to be paid to a possible tanning effect of the protein of the feed with lignosulfonates. The physical structure and behaviour of clays (bentonite, sepiolite) could explain some of the effects on the digestibility of energy in non-ruminant animals through their modification of the water retention and the transit time within the gut of the animal, and also on nitrogen utilization by ruminant animals.

MELCION J.P., 1995. Emploi des liants pour le pressage des aliments des animaux : aspects technologiques et nutritionnels. INRA Prod. Anim., 8 (2), 83-96. 